\section{Alkalose, respiratorische}

O. Müller-Plathe

Hamburg, Deutschland

\section{Englischer Begriff respiratory alkalosis}

Definition Die respiratorische Alkalose ist die durch Verminderung von Kohlendioxid (Hypokapnie) infolge alveolärer Hyperventilation entstehende Störung des Säure-BasenGleichgewichts.

Beschreibung Allen respiratorischen Alkalosen gemeinsam sind die Abnahme von $p \mathrm{CO}_{2}$ ( $\triangleright$ Kohlendioxidpartialdruck) und ein $\mathrm{pH}$-Wert-Anstieg, der je nach dem Grad der Kompensation unterschiedlich stark ausgeprägt ist.

Bei der akuten respiratorischen Alkalose (z. B. Hyperventilationssyndrom) besteht ein starker $\mathrm{pH}$-Wert-Anstieg, denn es findet lediglich Pufferung durch die Nichtbicarbonatpuffer statt, wobei Bicarbonat im Wesentlichen auf Kosten von $\mathrm{H}-\mathrm{Hb} \mathrm{zu} \mathrm{H}_{2} \mathrm{CO}_{3}$ umgewandelt wird. Es resultiert nur eine geringfügige Abnahme von $c \mathrm{HCO}_{3}{ }^{-}$bei zunächst unveränderter Basenabweichung. Nach einigen Minuten heftiger Hyperventilation kann die Basenabweichung durch vermehrte Laktatproduktion infolge gesteigerter Glykolyse geringfügig abfallen.

Bei der chronischen respiratorischen Alkalose (z. B. Höhenatmung) tritt als Kompensation eine vermehrte renale Bicarbonatausscheidung hinzu ( $>$ Säureausscheidung, renale). ${ }_{c} \mathrm{HCO}_{3}{ }^{-}$und Basenabweichung nehmen deutlich ab. $\mathrm{pH}$ ändert sich relativ wenig. Die Entscheidung, ob die Störung in normaler Weise kompensiert ist oder nicht, kann mithilfe des Dia- gramms im Stichwort $>$ Säure-Basen-Stoffwechsel getroffen werden.

Vorkommen bei folgenden Störungen:

- Stimulation des Atemzentrums: Hyperventilationssyndrom durch Erregung; Störung des Atemzentrums durch Tumor, Subarachnoidalblutung, Meningitis, Enzephalitis, Hirntrauma; hormonale Einflüsse (Progesteron, Schwangerschaft); Medikamente (Theophyllin, Salizylate, Katecholamine, Analeptika); Leberzirrhose, septischer Schock (gramnegative Erreger)

- Reflektorische Stimulation: Höhenatmung, Lungenerkrankungen mit Hypoxämie, Lungenembolie, Atelektasen, Rechts-links-Shunt bei angeborenen Herzfehlern

- Mechanische Beatmung: artefizielle Hyperventilation

Auswirkungen Steigerung der neuromuskulären Erregbarkeit durch Abnahme der ionisierten Calciumfraktion im Plasma ( $\triangleright$ Calcium), evtl. mit Krämpfen, Parästhesien, Bronchiolenspasmus, typischen EKG-Veränderungen und Rhythmusstörungen. Abnahme der Hirndurchblutung, evtl. mit Schwindel und Bewusstseinsstörung, im Extremfall Enzephalomalazie. Zunahme der Sauerstoffaffinität des Hämoglobins mit Nachteilen für den $>$ Sauerstofftransport. Tendenz zur Hypokaliämie und Hyperchlorämie.

\section{Literatur}

Foster GT, Vaziri ND, Sassoons CS (2001) Respiratory alkalosis. Respir Care 46:384-391 